\title{
Existence of nontrivial solution for a nonlocal problem with subcritical nonlinearity
}

Jing Zhang ${ }^{1 *}$ and Zhongyi Zhang ${ }^{2}$ (D)

${ }^{\text {*Correspondence: }}$
jinshizhangjing@163.com
${ }^{1}$ Mathematics Sciences College,
Inner Mongolia Normal University,
Hohhot, P.R. China
Full list of author information is
available at the end of the article

\section{Abstract}

In this paper, we consider the following new nonlocal Dirichlet boundary value problem:

$$
\begin{cases}-\left(a-b \int_{\Omega}|\nabla u|^{2} d x\right) \Delta u=\lambda u+g(x, u), & x \in \Omega, \\ u=0, & x \in \partial \Omega,\end{cases}
$$

where $a$ and $b$ are positive, $\lambda$ is a positive parameter, $0 \leq \lambda<a \lambda_{1}, \lambda_{1}$ is the first eigenvalue of operator $-\Delta$. Under appropriate assumptions on the function $g$ which is of subcritical growth, we obtain a nontrivial solution.

MSC: Primary 35B33; secondary 35B38; 35B09

Keywords: Nonlocal problem; Nontrivial solution; Subcritical nonlinearity

\section{Introduction and main result}

In this paper, we consider the following new nonlocal Dirichlet boundary value problem:

$$
\begin{cases}-\left(a-b \int_{\Omega}|\nabla u|^{2} d x\right) \Delta u=\lambda u+g(x, u), & x \in \Omega, \\ u=0, & x \in \partial \Omega,\end{cases}
$$

where $a$ and $b$ are positive, $\lambda$ is a positive parameter.

The search for a nontrivial solution of problem (1.1) is a new subject and of great significance. We put forward a new nonlocal term $a-b \int_{\Omega}|\nabla u|^{2} d x$, which is different from the well known nonlocal term $a+b \int_{\Omega}|\nabla u|^{2} d x$ and presents a lot of interesting difficulties.

Recently, mathematical studies have focused on the existence of solutions of the Kirchhoff type problem

$$
\begin{cases}-\left(a+b \int_{\Omega}|\nabla u|^{2} d x\right) \Delta u=g(x, u), & x \in \Omega, \\ u=0, & x \in \partial \Omega,\end{cases}
$$

(c) The Author(s) 2018. This article is distributed under the terms of the Creative Commons Attribution 4.0 International License (http://creativecommons.org/licenses/by/4.0/), which permits unrestricted use, distribution, and reproduction in any medium, provided you give appropriate credit to the original author(s) and the source, provide a link to the Creative Commons license, and indicate if changes were made. 
where $a>0, b>0$ and $\Omega$ is either a smooth bounded domain in $\mathbb{R}^{N}$ or $\Omega=\mathbb{R}^{N}$. The results about problem with subcritical nonlinearity can be seen in [1-5] and the critical cases in [6-13]. Here we do not present the results in detail, someone who is interested in them can consult the references therein.

However, there are only few results about problem (1.1). When $\lambda=0$ and $g(x, u)=|u|^{p-2} u$ was of subcritical growth, Yin and Liu [14] considered

$$
\begin{cases}-\left(a-b \int_{\Omega}|\nabla u|^{2} d x\right) \Delta u=|u|^{p-2} u, & x \in \Omega, \\ u=0, & x \in \partial \Omega\end{cases}
$$

and obtained existence and multiplicity of nontrivial solutions. When $\lambda=0$ and $g(x, u)=$ $f_{\lambda}(x)|u|^{p-2} u$, Lei [15] considered

$$
\begin{cases}-\left(a-b \int_{\Omega}|\nabla u|^{2} d x\right) \Delta u=f_{\lambda}(x)|u|^{p-2} u, & x \in \Omega, \\ u=0, & x \in \partial \Omega .\end{cases}
$$

Under some special conditions and for $1<p<2$, the author obtained two solutions. Lei [16] also investigated

$$
\begin{cases}-\left(a-b \int_{\Omega}|\nabla u|^{2} d x\right) \Delta u=\frac{\lambda}{u^{\gamma}}, & x \in \Omega, \\ u=0, & x \in \partial \Omega\end{cases}
$$

and, when $0<\gamma<1$ and $0<\lambda<\lambda_{*}$, at least two positive solutions were obtained. Wang [17] studied a nonlocal problem involving critical exponent, namely

$$
\left\{\begin{array}{l}
-\left(a-b \int_{\Omega}|\nabla u|^{2} d x\right) \Delta u=|u|^{2} u+\mu f(x), \quad x \in \mathbb{R}^{4}, \\
u \in D^{1,2}\left(\mathbb{R}^{4}\right)
\end{array}\right.
$$

for which infinitely many positive solutions and at least two positive solutions were found for $\mu=0$ and $\mu \in\left(0, \mu_{*}\right]$. For some other important results the interested reader is also referred to [18-21].

We are inspired by the above articles and consider a new problem which is different from the mentioned above. Assume that nonlinearity $g$ satisfies the following assumptions:

$\left(g_{1}\right) g$ is continuous, $1 \leq i \leq N,|g(x, u)| \leq C\left(1+|u|^{p-1}\right)$ for some $C>0$ and $2<p<2^{*}$, where $2^{*}=\frac{2 N}{N-2}$ if $N \geq 3,2^{*}=\infty$ if $N=1$ or 2 ;

$\left(g_{2}\right) g(x, u)=o(u)$ uniformly in $x$ as $u \rightarrow 0$;

$\left(g_{3}\right) u \mapsto \frac{g(x, u)}{u}$ is positive for $u \neq 0$, nonincreasing on $(-\infty, 0)$ and nondecreasing on $(0,+\infty)$.

Now, we state our main result.

Theorem 1.1 Suppose that conditions $\left(g_{1}\right)-\left(g_{3}\right)$ and $0 \leq \lambda<a \lambda_{1}$ hold, then problem (1.1) has a nontrivial solution. 


\section{Preliminary results}

In this section, we present the variational results which will be used in the proof of Theorem 1.1. Let $E:=H_{0}^{1}(\Omega)$ be endowed with the usual norm

$$
\|u\|=\langle u, u\rangle^{1 / 2}=\left(\int_{\Omega}|\nabla u|^{2}\right)^{1 / 2}
$$

The usual norm in the Lebesgue space $L^{p}(\Omega)$ is denoted by $|u|_{p}$.

A function $u \in E$ is called a weak solution of problem (1.1) if

$$
a \int_{\Omega} \nabla u \nabla v d x-b\|u\|^{2} \int_{\Omega} \nabla u \nabla v d x=\lambda \int_{\Omega} u v d x-\int_{\Omega} g(x, u) v d x, \quad \forall v \in E .
$$

Moreover, our assumptions imply that the solutions of (1.1) are the critical points of the functional defined in $E$ by

$$
I(u)=\frac{a}{2}\|u\|^{2}-\frac{b}{4}\|u\|^{4}-\frac{\lambda}{2} \int_{\Omega}|u|^{2} d x-\int_{\Omega} G(x, u) d x .
$$

It is easy to see for $\forall u, v \in E$,

$$
\left\langle I^{\prime}(u), v\right\rangle=a \int_{\Omega} \nabla u \nabla v d x-b\|u\|^{2} \int_{\Omega} \nabla u \nabla v d x-\lambda \int_{\Omega} u v d x-\int_{\Omega} g(x, u) v d x .
$$

Let $\lambda_{i}(i=1,2, \ldots)$ be the eigenvalues of operator $-\Delta$ with zero Dirichlet boundary condition. It is well known that each eigenvalue $\lambda_{i}$ is positive, isolated and has finite multiplicity, the smallest eigenvalue $\lambda_{1}$ being simple and $\lambda_{i} \rightarrow \infty$ as $i \rightarrow \infty$. Here we only need the first eigenvalue of $-\Delta$, where $\lambda_{1}=\inf _{u \neq 0} \frac{\int_{\Omega}|\nabla u|^{2}}{\int_{\Omega}|u|^{2}}$ and assume that $0 \leq \lambda<a \lambda_{1}$.

\section{Proof of Theorem 1.1}

In this section, we will prove Theorem 1.1, so from now on we always suppose that $\left(g_{1}\right)$ $\left(g_{3}\right)$ hold. First, $\left(g_{1}\right)$ and $\left(g_{2}\right)$ imply that for each $\varepsilon>0$ there is a $C_{\varepsilon}>0$ such that

$$
|g(x, u)| \leq \varepsilon|u|+C_{\varepsilon}|u|^{p-1} \quad \text { for all } u \in \mathbb{R} .
$$

And using $\left(g_{2}\right)$ and $\left(g_{3}\right)$, one can easily check that

$$
G(x, u) \geq 0 \quad \text { and } \quad g(x, u) u \geq 2 G(x, u)>0 \quad \text { if } u \neq 0 .
$$

Lemma 3.1 If $0 \leq \lambda<a \lambda_{1}$, then there exists a sequence $\left\{u_{n}\right\} \subset E$ satisfying $I\left(u_{n}\right) \rightarrow c$, $I^{\prime}\left(u_{n}\right) \rightarrow 0$, where $0<c<\frac{a^{2}}{4 b}$.

Proof For $\lambda_{1}=\inf _{u \neq 0} \frac{\int_{\Omega}|\nabla u|^{2}}{\int_{\Omega}|u|^{2}}$, then

$$
\left(a-\frac{\lambda}{\lambda_{1}}\right) \int_{\Omega}|\nabla u|^{2} \leq a \int_{\Omega}|\nabla u|^{2}-\lambda \int_{\Omega}|u|^{2} \leq a \int_{\Omega}|\nabla u|^{2} .
$$


Also by (3.1), we can choose a sufficiently small $\varepsilon=\frac{\lambda_{1}}{2}\left(a-\frac{\lambda}{\lambda_{1}}\right)$, and then

$$
\begin{aligned}
I(u) & =\frac{a}{2}\|u\|^{2}-\frac{b}{4}\|u\|^{4}-\frac{\lambda}{2} \int_{\Omega}|u|^{2}-\int_{\Omega} G(x, u) \\
& \geq \frac{1}{2}\left(a-\frac{\lambda}{\lambda_{1}}\right) \int_{\Omega}|\nabla u|^{2}-\frac{b}{4}\left(\int_{\Omega}|\nabla u|^{2}\right)^{2}-\frac{\varepsilon}{2} \int_{\Omega}|u|^{2}-\frac{C_{\varepsilon}}{p} \int_{\Omega}|u|^{p} \\
& \geq \frac{1}{2}\left(a-\frac{\lambda}{\lambda_{1}}\right) \int_{\Omega}|\nabla u|^{2}-\frac{b}{4}\|u\|^{4}-\frac{\varepsilon}{2 \lambda_{1}} \int_{\Omega}|\nabla u|^{2}-\frac{C_{1} C_{\varepsilon}}{p}\|u\|^{p} \\
& \geq \frac{1}{4}\left(a-\frac{\lambda}{\lambda_{1}}\right)\|u\|^{2}-\frac{b}{4}\|u\|^{4}-\frac{C_{1} C_{\varepsilon}}{p}\|u\|^{p},
\end{aligned}
$$

Since $4<p<2^{*}$, for small enough $\rho>0$, for all $u \in E$ and $\|u\|=\rho$, it holds that $I(u)=\gamma>0$. On the other hand, for $u \neq 0$ and $t \in \mathbb{R}$,

$$
I(t u)=\frac{a t^{2}}{2}\|u\|^{2}-\frac{b t^{4}}{4}\|u\|^{4}-\frac{\lambda t^{2}}{2} \int_{\Omega}|u|^{2}-\int_{\Omega} G(x, t u),
$$

so that when $t \rightarrow \infty$, we have $I(t u) \rightarrow-\infty$. This means that there is a $t_{1}$ such that $u_{1}=$ $t_{1} u \in E,\left\|u_{1}\right\|>\rho$ and $I\left(u_{1}\right)<0$. As a consequence, by the mountain pass lemma without (PS) condition [22], there exists a sequence $\left\{u_{n}\right\} \subset E$ such that $I\left(u_{n}\right) \rightarrow c, I^{\prime}\left(u_{n}\right) \rightarrow 0$ for

$$
c=\inf _{h \in \Gamma} \max _{u \in h([0,1])} I(u) \geq \gamma>0
$$

where

$$
\Gamma=\left\{h \in C([0,1], E): h(0)=0, h(1)=u_{1}\right\}
$$

Because

$$
\begin{aligned}
\max _{t \in[0,1]} I\left(t u_{1}\right) & =\max _{t \in[0,1]}\left\{\frac{a t^{2}}{2}\left\|u_{1}\right\|^{2}-\frac{b t^{4}}{4}\left\|u_{1}\right\|^{4}-\frac{\lambda t^{2}}{2} \int_{\Omega}\left|u_{1}\right|^{2}-\int_{\Omega} G\left(x, t u_{1}\right)\right\} \\
& <\max _{t \in[0,1]}\left\{\frac{a t^{2}}{2}\left\|u_{1}\right\|^{2}-\frac{b t^{4}}{4}\left\|u_{1}\right\|^{4}\right\} \\
& \leq \frac{a^{2}}{4 b},
\end{aligned}
$$

it is easy to obtain that $0<c<\frac{a^{2}}{4 b}$ according to the definition of $c$.

Lemma 3.2 Under the condition $c<\frac{a^{2}}{4 b}$, I satisfies the $(P S)_{c}$ condition, i.e., any $(P S)_{c}$ sequence of I has a convergent subsequence.

Proof We drew on the experience of [14]. Let $\left\{u_{n}\right\} \subset E$ be such that $I\left(u_{n}\right) \rightarrow c, I^{\prime}\left(u_{n}\right) \rightarrow 0$.

Since by (3.2)

$$
\begin{aligned}
c+o(1) & =I\left(u_{n}\right)-\frac{1}{2}\left\langle I^{\prime}\left(u_{n}\right), u_{n}\right\rangle \\
& =\frac{a}{2}\left\|u_{n}\right\|^{2}-\frac{b}{4}\left\|u_{n}\right\|^{4}-\frac{\lambda}{2} \int_{\Omega}\left|u_{n}\right|^{2}-\int_{\Omega} G\left(x, u_{n}\right)
\end{aligned}
$$


Chang and Chang Advances in Difference Equations ( 2018) 2018:359

Page 5 of 8

$$
\begin{aligned}
& -\left[\frac{a}{2}\left\|u_{n}\right\|^{2}-\frac{b}{2}\left\|u_{n}\right\|^{4}-\frac{\lambda}{2} \int_{\Omega}\left|u_{n}\right|^{2}-\frac{1}{2} g\left(x, u_{n}\right)\right] \\
\geq & \frac{b}{4}\left\|u_{n}\right\|^{4},
\end{aligned}
$$

we know that $\left\{u_{n}\right\}$ is bounded in $E$. By passing to a subsequence, still denoted $\left\{u_{n}\right\}$, we may assume that there is a $u \in E$ such that

$$
\begin{aligned}
& u_{n} \rightarrow u \quad \text { in } E, \\
& u_{n} \rightarrow u \quad \text { in } L^{s}(\Omega) \text { for } s \in\left[1,2^{*}\right), \\
& u_{n}(x) \rightarrow u(x) \quad \text { for a.e. } x \in \Omega .
\end{aligned}
$$

On account of

$$
\begin{aligned}
o(1) & =\left\langle I^{\prime}\left(u_{n}\right), u_{n}-u\right\rangle \\
& =\left(a-b\left\|u_{n}\right\|^{2}\right) \int_{\Omega} \nabla u_{n} \nabla\left(u_{n}-u\right)-\lambda \int_{\Omega} u_{n}\left(u_{n}-u\right)-\int_{\Omega} g\left(x, u_{n}\right)\left(u_{n}-u\right)
\end{aligned}
$$

and

$$
\left|\int_{\Omega} u_{n}\left(u_{n}-u\right)\right| \leq\left(\int_{\Omega}\left|u_{n}\right|^{2}\right)^{\frac{1}{2}}\left(\int_{\Omega}\left|u_{n}-u\right|^{2}\right)^{\frac{1}{2}}
$$

also by (3.1)

$$
\begin{aligned}
& \left|\int_{\Omega} g\left(x, u_{n}\right)\left(u_{n}-u\right)\right| \\
& \quad \leq \varepsilon\left|\int_{\Omega} u_{n}\left(u_{n}-u\right)\right|+\left.C_{\varepsilon}\left|\int_{\Omega}\right| u_{n}\right|^{p-2} u_{n}\left(u_{n}-u\right) \mid \\
& \quad \leq \varepsilon\left(\int_{\Omega}\left|u_{n}\right|^{2}\right)^{\frac{1}{2}}\left(\int_{\Omega}\left|u_{n}-u\right|^{2}\right)^{\frac{1}{2}}+C_{\varepsilon}\left(\int_{\Omega}\left(\left|u_{n}\right|^{p-1}\right)^{\frac{p}{p-1}}\right)^{\frac{p-1}{p}}\left(\int_{\Omega}\left(\left|u_{n}-u\right|^{p}\right)\right)^{\frac{1}{p}},
\end{aligned}
$$

because $u_{n} \rightarrow u$ in $L^{s}(\Omega), s \in\left[1,2^{*}\right)$, the above two formulas show that when $n \rightarrow \infty$,

$$
\left(a-b\left\|u_{n}\right\|^{2}\right) \int_{\Omega} \nabla u_{n} \nabla\left(u_{n}-u\right) \rightarrow 0
$$

If there exists a subsequence of $\left\{u_{n}\right\}$, still denoted $\left\{u_{n}\right\}$, satisfying $\left\|u_{n}\right\|^{2} \rightarrow \frac{a}{b}$, define a functional

$$
\varphi(u)=\frac{\lambda}{2} \int_{\Omega}|u|^{2}+\int_{\Omega} G(x, u), \quad u \in E .
$$

Then

$$
\left\langle\varphi^{\prime}(u), v\right\rangle=\lambda \int_{\Omega} u v+\int_{\Omega} g(x, u) v, \quad u, v \in E,
$$

and

$$
\left\langle\varphi^{\prime}\left(u_{n}\right)-\varphi^{\prime}(u), v\right\rangle=\lambda \int_{\Omega}\left(u_{n}-u\right) v+\int_{\Omega}\left[g\left(x, u_{n}\right)-g(x, u)\right] v .
$$


Claim. $\left\langle\varphi^{\prime}\left(u_{n}\right)-\varphi^{\prime}(u), v\right\rangle \rightarrow 0, \forall v \in E$.

Firstly,

$$
\lambda \int_{\Omega}\left(u_{n}-u\right) v \leq \lambda\left(\int_{\Omega}\left|u_{n}-u\right|^{2}\right)^{\frac{1}{2}}\left(\int_{\Omega}|v|^{2}\right)^{\frac{1}{2}}
$$

since $u_{n} \rightarrow u$ in $L^{2}(\Omega)$, thus $\lambda \int_{\Omega}\left(u_{n}-u\right) v \rightarrow 0$.

Secondly, to prove the claim, we only need to prove

$$
\lim _{n \rightarrow \infty} \int_{\Omega}\left|g\left(x, u_{n}\right)-g(x, u)\right||v|=0 .
$$

If (3.4) is not true, then there exist a constant $\varepsilon_{0}>0$ and a subsequence $u_{k_{i}}$ such that

$$
\int_{\Omega}\left|g\left(x, u_{k_{i}}\right)-g(x, u)\right||v| \geq \varepsilon_{0}, \quad \forall i \in \mathbb{N}
$$

Since $u_{n} \rightarrow u$ in $L^{p}(\Omega)$, passing to a subsequence if necessary, we can assume that $\sum_{i=1}^{\infty}\left|u_{k_{i}}-u\right|_{p}^{p}<+\infty$. Set

$$
\omega(x)=\left[\sum_{i=1}^{\infty}\left|u_{k_{i}}(x)-u(x)\right|^{p}\right]^{\frac{1}{p}}, \quad \forall x \in \Omega .
$$

Then $\omega \in L^{p}(\Omega)$. Note that for $\forall i \in \mathbb{N}, x \in \Omega$,

$$
\begin{aligned}
\left|g\left(x, u_{k_{i}}\right)-g(x, u)\right||v| \\
\quad \leq\left(\left|g\left(x, u_{k_{i}}\right)\right|+|g(x, u)|\right)|v| \\
\quad \leq\left[\varepsilon\left(\left|u_{k_{i}}\right|+|u|\right)+C_{\varepsilon}\left(\left|u_{k_{i}}\right|^{p-1}+|u|^{p-1}\right)\right]|v| \\
\quad \leq\left[2^{2} \varepsilon\left(\left|u_{k_{i}}-u\right|+|u|\right)+2^{p} C_{\varepsilon}\left(\left|u_{k_{i}}-u\right|^{p-1}+|u|^{p-1}\right)\right]|v| \\
\quad \leq\left[2^{2} \varepsilon(|\omega|+|u|)+2^{p} C_{\varepsilon}\left(|\omega|^{p-1}+|u|^{p-1}\right)\right]|v| \\
\quad:=f(x),
\end{aligned}
$$

and

$$
\begin{aligned}
\int_{\Omega} f(x) d x & =\int_{\Omega}\left[2^{2} \varepsilon(|\omega|+|u|)+2^{p} C_{\varepsilon}\left(|\omega|^{p-1}+|u|^{p-1}\right)\right]|v| \\
& \leq 2^{2} \varepsilon\left(|\omega|_{2}+|u|_{2}\right)|v|_{2}+2^{p} C_{\varepsilon}\left(|\omega|_{p}^{p-1}+|u|_{p}^{p-1}\right)|v|_{p}<+\infty
\end{aligned}
$$

Since $u_{k_{i}} \rightarrow u$ a.e. in $\Omega$, then by (3.6), (3.7) and Lebesgue Dominated Convergence Theorem, we have

$$
\lim _{i \rightarrow \infty} \int_{\Omega}\left|g\left(x, u_{k_{i}}(x)\right)-g(x, u(x))\right||v|=0,
$$


which contradicts (3.5). Hence (3.4) holds. Then the claim follows. By arbitrariness of $v$, then

$$
\left\|\varphi^{\prime}\left(u_{n}\right)-\varphi^{\prime}(u)\right\|_{E^{\prime}} \rightarrow 0
$$

and $\varphi^{\prime}\left(u_{n}\right) \rightarrow \varphi^{\prime}(u)$ in $E^{\prime}$. While $\left\langle I^{\prime}\left(u_{n}\right), v\right\rangle=\left(a-b\left\|u_{n}\right\|^{2}\right)\left\langle u_{n}, v\right\rangle-\left\langle\varphi^{\prime}\left(u_{n}\right), v\right\rangle,\left\langle I^{\prime}\left(u_{n}\right), v\right\rangle \rightarrow 0$, $a-b\left\|u_{n}\right\|^{2} \rightarrow 0$, hence $\varphi^{\prime}\left(u_{n}\right) \rightarrow 0$, i.e.,

$$
\left\langle\varphi^{\prime}(u), v\right\rangle=\lambda \int_{\Omega} u v+\int_{\Omega} g(x, u) v=0, \quad \forall v \in E,
$$

and then we have

$$
\lambda u(x)+g(x, u(x))=0 \quad \text { for a.e. } x \in \Omega,
$$

by the fundamental lemma of the variational method (see [23]). It follows that $u=0$. So

$$
\varphi\left(u_{n}\right)=\frac{\lambda}{2} \int_{\Omega}\left|u_{n}\right|^{2}+\int_{\Omega} G\left(x, u_{n}\right) \rightarrow \frac{\lambda}{2} \int_{\Omega}|u|^{2}+\int_{\Omega} G(x, u)=0 .
$$

Hence we see that $I\left(u_{n}\right)=\frac{a}{2}\left\|u_{n}\right\|^{2}-\frac{b}{4}\left\|u_{n}\right\|^{4}-\frac{\lambda}{2} \int_{\Omega}\left|u_{n}\right|^{2}-\int_{\Omega} G\left(x, u_{n}\right) \rightarrow \frac{a^{2}}{4 b}$ from $\left\|u_{n}\right\|^{2} \rightarrow$ $\frac{a}{b}$. This is a contradiction to $I\left(u_{n}\right) \rightarrow c<\frac{a^{2}}{4 b}$. Then $a-b\left\|u_{n}\right\|^{2} \rightarrow 0$ is not true and any subsequence of $\left\{a-b\left\|u_{n}\right\|^{2} \rightarrow 0\right\}$ does not converge to zero. Therefore there exists a $\delta>0$ such that $\left|a-b\left\|u_{n}\right\|^{2}\right|>\delta>0$ when $n$ is large enough. It is clear that $\left\{a-b\left\|u_{n}\right\|^{2} \rightarrow 0\right\}$ is bounded. It follows from (3.3) that $\int_{\Omega} \nabla u_{n} \nabla\left(u_{n}-u\right) \rightarrow 0$. So $\left\|u_{n}\right\| \rightarrow\|u\|$. Hence $u_{n} \rightarrow u$ in $E$ due to the uniform convexity of $E$.

Proof of Theorem 1.1 According to Lemma 3.1, there exists a sequence $\left\{u_{n}\right\} \in E$ satisfying $I\left(u_{n}\right) \rightarrow c>0, I^{\prime}\left(u_{n}\right) \rightarrow 0$. By Lemma 3.2, $\left\{u_{n}\right\}$, which is the sequence obtained by Lemma 3.1, possesses a convergent to $u$ subsequence (still denoted by $\left\{u_{n}\right\}$ ). So it follows from the continuity that $I\left(u_{n}\right) \rightarrow c>0, I^{\prime}\left(u_{n}\right) \rightarrow 0$. But $I(0)=0$, therefore $u \neq 0$, that is, $u$ is a nontrivial solution of problem (1.1).

\begin{abstract}
Acknowledgements
We would like to thank the referee for his/her valuable comments and helpful suggestions which have led to an improvement of the presentation of this paper. The authors are supported by The Inner Mongolia Autonomous Region university scientific research project (NJZY18021) and Postdoctoral research project of Inner Mongolia University (21100-5175504) and Inner Mongolia Normal University introduces high-level scientific research projects (2016YJRC005) and Research project of Inner Mongolia Normal University (2016ZRYB001).
\end{abstract}

Funding

Not applicable.

Availability of data and materials

Not applicable.

Competing interests

The authors declare that they have no competing interests.

Authors' contributions

All authors contributed equally to the manuscript. All authors read and approved the final manuscript.

\title{
Author details
}

${ }^{1}$ Mathematics Sciences College, Inner Mongolia Normal University, Hohhot, P.R. China. ${ }^{2}$ College of Mathematics,

Changchun Normal University, Changchun, P.R. China. 


\section{Publisher's Note}

Springer Nature remains neutral with regard to jurisdictional claims in published maps and institutional affiliations.

Received: 4 August 2018 Accepted: 28 September 2018 Published online: 10 October 2018

\section{References}

1. Batkam, C.-J:: An elliptic equation under the effect of two nonlocal terms. Math. Methods Appl. Sci. 39, 1535-1547 (2016)

2. Delgado, M., Figueiredo, G.-M., Gayte, I., Morales-Rodrigo, C.: An optimal control problem for a Kirchhoff-type equation. ESAIM Control Optim. Calc. Var. 23, 773-790 (2017)

3. Ding, L., Meng, Y.J., Xiao, S.W., Zhang, J.L.: Existence of two positive solutions for indefinite Kirchhoff equations in $\mathbb{R}^{3}$. Electron. J. Differ. Equ. 2016, 35 (2016)

4. Mao, A.M., Zhu, X.C.: Existence and multiplicity results for Kirchhoff problems. Mediterr. J. Math. 14, 58 (2017)

5. Tang, X.H., Cheng, B.T.: Ground state sign-changing solutions for Kirchhoff type problems in bounded domains. J. Differ. Equ. 261, 2384-2402 (2016)

6. He, X., Zou, W.: Existence and concentration behavior of positive solutions for a Kirchhoff equation in $\mathbb{R}^{3}$. J. Differ. Equ. $252,1813-1834(2012)$

7. Liu, Z., Guo, S.J., Fang, Y.Q.: Positive solutions of Kirchhoff type elliptic equations in $\mathbb{R}^{4}$ with critical growth. Math. Nachr. 290, 367-381 (2016)

8. Peng, C.Q.: The existence and concentration of ground-state solutions for a class of Kirchhoff type problems in $\mathbb{R}^{3}$ involving critical Sobolev exponents. Bound. Value Probl. 64, 64 (2017)

9. $\mathrm{Wu}, \mathrm{X}$ : Existence of nontrivial solutions and hingh energy solutions for Schrödinger-Kirchhoff-type equations in $\mathbb{R}^{N}$. Nonlinear Anal. 12, 1278-1287 (2011)

10. Yang, L., Liu, Z.S., Ouyang, Z.: Multiplicity results for the Kirchhoff type equations with critical growth. Appl. Math. Lett. 63, 118-123 (2017)

11. Zhang, J.: The Kirchhoff type Schrödinger problem with critical growth. Nonlinear Anal., Real World Appl. 28, 153-170 (2016)

12. Zhang, J.: The critical Neumann problem of Kirchhoff type. Appl. Math. Comput. 274, 519-530 (2016)

13. Zhang, C.H., Liu, Z.S.: Multiplicity of nontrivial solutions for a critical degenerate Kirchhoff type problem. Appl. Math. Lett. 69, 87-93 (2017)

14. Yin, G.S., Liu, J.S.: Existence and multiplicity of nontrivial solutions for a nonlocal problem. Bound. Value Probl. 2015 26 (2015)

15. Lei, C.Y., Liao, J.F., Suo, H.M.: Multiple positive solutions for a class of nonlocal problems involving a sign-changing potential. Electron. J. Differ. Equ. 9, 1 (2017)

16. Lei, C.Y., Chu, C.M., Suo, H.M.: Positive solutions for a nonlocal problem with singularity. Electron. J. Differ. Equ. 85 , (2017)

17. Wang, Y., Suo, H.M., Lei, C.Y.: Multiple positive solutions for a nonlocal problem involving critical exponent. Electron. J. Differ. Equ. 275, 1 (2017)

18. Liang, S., Shi, S.: Soliton solutions to Kirchhoff type problems involving the critical growth in $\mathbb{R}^{N}$. Nonlinear Anal. 81, 31-41 (2013)

19. Liang, S., Zhang, J.: Existence of solutions for Kirchhoff type problems with critical nonlinearity in $\mathbb{R}^{3}$. Nonlinear Anal., Real World Appl. 17, 126-136 (2014)

20. Liang, S., Zhang, J.: Multiplicity of solutions for the noncooperative Schrödinger-Kirchhoff system involving the fractional $p$-Laplacian in $\mathbb{R}^{N}$. Z. Angew. Math. Phys. 68, 63 (2017)

21. Zhang, B.: Multi-peak solutions for a nonlinear Schrödinger-Poisson system including critical growth in $\mathbb{R}^{3}$. Adv. Differ. Equ. 2016, 189 (2016)

22. Ambrosetti, A., Rabinowitz, P.H.: Dual variational methods in critical points theory and applications. J. Funct. Anal. 14, 349-381 (1973)

23. Lu, W.D.: The Variational Method in Differential Equation. Sichuan University Press, Sichuan (1995)

\section{Submit your manuscript to a SpringerOpen ${ }^{\circ}$ journal and benefit from:}

- Convenient online submission

- Rigorous peer review

- Open access: articles freely available online

- High visibility within the field

- Retaining the copyright to your article

Submit your next manuscript at $\downarrow$ springeropen.com 